\title{
Innovación docente en estudios de patrimonio: clase invertida y creación de contenidos multimedia en el Grado de Historia del Arte de la Universidad de Oviedo
}

\section{Educational innovation in Heritage studies: flipped classroom and media creation in the History of Art degree at the University of Oviedo}

\author{
Noelia Fernández García1 \& María Pilar García Cuetos²
}

Fecha de recepción: 30/09/2020; Fecha de revisión21/10/2020; Fecha de aceptación: 11/02/2021

Cómo citar este artículo:

Fernández García, N. \& García Cuetos, M.P. (2021). Innovación docente en estudios de patrimonio: clase invertida y creación de contenidos multimedia en el Grado de Historia del Arte de la Universidad de Oviedo. Revista de Innovación y Buenas Prácticas Docentes, 10(1), 51-62.

Autor de Correspondencia: fernandezgnoelia@uniovi.es

\begin{abstract}
Resumen:
Este trabajo recoge los objetivos, desarrollo, resultados y conclusiones del proyecto de innovación docente "La clase inversa y el podcast como herramientas de aprendizaje colaborativo en las asignaturas de Historia de la Conservación y Restauración del Patrimonio Histórico-Artístico y Gestión y legislación del Patrimonio Histórico-Artístico (Grado de Historia del Arte)" desarrollado en el Departamento de Historia del Arte y Musicología de la Universidad de Oviedo durante el primer semestre del curso 2019-2020.

A partir del método de la clase inversa y el manejo de las TICs se ha conseguido no sólo un a mejora en los resultados de evaluación en ambas asignaturas y un aumento en las competencias relativas a las herramientas audiovisuales, muy ventajosas en la futura profesionalización del alumnado, sino también una mayor participación del mismo en su proceso de aprendizaje y en el trabajo colectivo. No obstante, Ia valoración final de los resultados del proyecto, por parte de alumnado y docentes, indica la necesid ad de ajustar estas iniciativas a la realidad académica y la carga de trabajo que suponen.
\end{abstract}

Palabras clave: Autoaprendizaje, Historia del Arte Método audiovisual, trabajo en equipo, Universidad

\begin{abstract}
:
This article aims to present the objectives, development, results and conclusions achieved by the teaching innovation project "Flipped Classroom and podcast as cooperative learning tools within the subjects 'History of Preservation and Restoration of Historical-Artistic Heritage' and 'Management and Legislation of Historical-Artistic Heritage"' developed in the Department of Art History and Music Sciences at the University of Oviedo during the first term of the academic year 2019-2020.

The implementation of the Flipped Classroom method together with the use of ICTs have made possible not only an improvement in the evaluation results and in the abilities related to audio-visual tools, remarkably advantageous for students' professional future, but also a higher level of participation in their self-learning process and cooperative work. Nevertheless, the final assessment of the achieved results by both students and teachers state the need of adjusting these experiences to the academic reality and the workload they imply.
\end{abstract}

Key Words: Audio-visual method, cooperative work, History of Art, self-learning, University

\footnotetext{
' Universidad de Oviedo (España), fernandezgnoelia@uniovi.es; CÓDIGO ORCID: https://orcid.org/00000001-5675-0642

${ }^{2}$ Universidad de Oviedo (España),g guetos@uniovi.es; CÓDIGO ORCID: https://orcid.org/0000-00031221-5853
} 


\section{INTRODUCCIÓN}

El proyecto de innovación presentado en este trabajo se corresponde con la segunda fase del realizado durante el curso 2018/2019 bajo el título "La clase inversa y el viaje docente al servicio de la renovación de la asignatura de Historia de la Conservación y Restauración del Patrimonio Histórico-Artístico del Grado de Historia del Arte" en la Universidad de Oviedo. Para el desarrollo de este primer proyecto se decidió tomar metodología de la clase invertida como el punto de partida para lograr lo que los autores Bergmann y Sams (2014) denominan "aprendizaje invertido", el cual definen como "una estrategia pedagógica en la cual la enseñanza directa se mueve del espacio del aprendizaje grupal al espacio de aprendizaje individual" (p. 6), y aplicarla en la mencionada asignatura del tercer curso del grado.

Si bien es verdad que hasta el momento esta metodología ha sido aplicada, en gran medida, dentro de los ámbitos de la enseñanza primaria y secundaria, su introducción en la universidad se ha venido realizando de forma progresiva en los últimos tiempos en diversos campos de estudio e instituciones, incluida la Universidad de Oviedo y en diferentes proyectos de innovación centrados en el aprendizaje activo por parte del alumnado. En caso del ámbito universitario, la aplicación de la clase inversa en las prácticas docentes se debe a que la percepción del profesorado parece siempre coincidir en que las clases magistrales, o expositivas, resultan demasiado pasivas y no dan lugar a un aprendizaje activo por parte del alumnado (Rutherford, 2016).

La clase inversa no es una propuesta metodológica de un solo procedimiento, sino que puede realizarse a través de varios. No obstante, la idea clave siempre es la misma: el alumnado trabaja en su casa con los materiales ofrecidos por las y los docentes mientras que en las horas lectivas, tras reflexionar sobre los nuevos conocimientos adquiridos, los ponen en práctica. Así, el alumnado utiliza este tiempo para llevar a cabo "otros procesos de adquisición y práctica de conocimientos", como manifiestan Touron y Santiago (2009, p. 209), contando con la figura docente como guía en los citados procesos.

Los buenos resultados alcanzados y la buena valoración por parte del alumnado tuvieron como consecuencia la petición de un nuevo proyecto de innovación docente para el curso 2019/2020, así como la inclusión de un nuevo grupo de alumnado en el mismo, concretamente, el correspondiente a la asignatura de Gestión y Legislación del Patrimonio Histórico Artístico, de cuarto curso del grado en Historia del Arte.

Para este nuevo proyecto, y debido a una dificultad sobrevenida que impedíala realización del viaje docente - contemplado en el proyecto del curso anterior -, se planteó una combinación de la implementación de la Flipped Classroom y de las TICs, con la intención de que el alumnado, además de convertirse en el agente principal del proceso de aprendizaje (Rutherford, 2016), aprovechase su habilidad para apropiarse de las TICs (Herrera-Batista, 2009, p.1) y las incorporase a sus actividades académicas, pretendiendo como resultado la consecución de aprendizajes significativos en el alumnado universitario y tomando como base el trabajo colaborativo y en red (Trujillo 2011, p. 71). Todo esto bajo la pretensión de crear un nuevo entorno 
relacional en el aula, donde el alumnado pudiese sentirse más implicado y protagonista en el proceso de enseñanza-aprendizaje (Trujillo 2011, 61), además de ser capaz de presentar la elaboración de su propio conocimiento a partir de la creación de vídeos sobre los contenidos tratados en ambas asignaturas.

Los citados contenidos fueron abordados mediante el análisis de casos concretos, los instrumentos internacionales y nacionales, programas específicos relacionados con la intervención sobre bienes histórico-artísticos, y los correspondientes análisis sobre la gestión y programas de tutela de diferentes ejemplos de patrimonio histórico-artístico. El alumnado, por su parte, debía desarrollar las competencias necesarias para favorecer la reflexión sobre las implicaciones tanto de los procedimientos de restauración y sus consecuencias, como la de diferentes políticas en materia de tutela, gestión y conservación del patrimonio histórico-artístico, estableciendo conclusiones y diagnósticos básicos acerca de la situación actual del mismo, y presentándolos a compañeros y compañeras, facilitando su manejo y comprensión a través de la herramienta del vídeo.

Finalmente, podemos destacar que el hecho de que el alumnado alcance competencias nuevas - o las desarrolle en mayor medida - en lo referido al manejo de las tecnologías de la información y la comunicación, también puede revertir positivamente en su futuro laboral (Salinas 2004, 3). Hemos de pensar que durante estos pasados años hemos estado asistiendo no sólo a la cada vez mayor divulgación de contenidos culturales sino también a la difusión de instituciones culturales y sus actividades a través de este medio tecnológico compartido en webs oficiales o canales institucionales o profesionales en redes sociales, por lo que, como mencionábamos, este primer acercamiento a la aplicación de la tecnología en su campo de estudio puede suponer una oportunidad en su proceso de profesionalización.

\section{DESARROLLO DE LA EXPERIENCIA DE INNOVACIÓN}

Las asignaturas incluidas en este proyecto de innovación docente se desarrollaron a lo largo del primer semestre del curso 2019/2020. A partir del inicio del curso, se comenzó a proporcionar diversos materiales al alumnado (cronograma de la asignatura, guías y esquemas de los contenidos teóricos, bibliografía, documentos internacionales, experiencias y proyectos, vídeos, documentales y los materiales que se manejaron en las prácticas de aula) a través del Campus Virtual, plataforma cuyo empleo facilitó también la inclusión y participación de los alumnos y alumnas matriculados a tiempo parcial o que no pudieron asistir a las sesiones prácticas o teóricas por diversas circunstancias.

Asimismo, se planteó que el alumnado realizase dos vídeos diferentes que fueron presentados en las sesiones prácticas indicadas en el cronograma y almacenados en el Campus Virtual de la Universidad de Oviedo, de forma que fuesen accesibles a todos los alumnos y alumnas de cada asignatura a partir de los recursos que la propia plataforma ofrece. En este caso, aunque en un principio se pensó en 
utilizar la herramienta "Podcast", finalmente se optó por la utilización de la herramienta "Blog", por su operatividad a la hora de enlazar los vídeos desde los canales p rivados del alumnado en la plataforma YouTube. Asimismo, esta herramienta ofrecía además la posibilidad de permitir diferentes comentarios de compañeros y compañeras, de forma que, de nuevo, se potenciase la capacidad de trabajo y formación autónoma del alumnado a través de la educación virtual en aquellos casos en que la asistencia física al aula no fuera posible.

En lo referente a la creación de los contenidos y vídeos, las dinámicas de trabajo fueron dos: de forma grupal o individual, optándose por la segunda opción preferentemente para aquellos alumnos o alumnas que no pudiesen asistir con regularidad a las sesiones presenciales y pretendiendo mantener así una dinámica constante de trabajo colaborativo con sus compañeros y compañeras. De esta manera, se pretendió favorecer la adquisición de destrezas como las capacidades de análisis y comunicación, la escucha activa, la crítica constructiva o la asertividad, fundamentales para el trabajo en equipo.

De acuerdo con las actividades formativas presenciales recogidas en las Guías Docentes de las asignaturas incluidas en el proyecto, éstas se estructuraban de la siguiente forma con unos objetivos concretos:

1) Clases expositivas: pretendiendo dejar atrás la pasividad en el aula, se buscó estimular la participación activa y crítica, así como la atención al alumnado. El material didáctico de apoyo necesario fue previamente almacenado a las sesiones en la plataforma Campus Virtual, of reciéndose las orientaciones oportunas para favorecer la autonomía respecto al trabajo. Contar con una síntesis de los temas favorece una participación más activa y realizar una lectura previa facilita una mejor comprensión de los contenidos expuestos.

2) Prácticas de aula: fueron propuestas como actividades relacionadas con los contenidos teóricos de las asignaturas y con otros aspectos transversales de la materia y se integraron de forma coherente con las sesiones teóricas, siendo resultado de una labor de planificación, programación y reflexión por parte de las docentes. Como apoyo a las prácticas, también se realizó una selección de materiales proporcionados previamente, aplicando también el método de la clase inversa.

3) Tutorías grupales: las cuatro tutorías grupales de cada asignatura, de carácter voluntario para el alumnado, fueron utilizadas para la presentación del proyecto por parte de las docentes $y$, durante el proceso de recogida y análisis de información y de creación de los vídeos, estas sesiones fueron de gran utilidad para presentar avances, consultar dudas o aportar sugerencias por parte del alumnado.

Las condiciones propuestas para nuestro alumnado en estas sesiones, principalmente en las clases expositivas y las prácticas de aula, fueron las siguientes:

- No apagar el teléfono móvil: este dispositivo puede ser utilizado para alentar la atención en clase si se utiliza como una herramienta que permite la búsqueda rápida de información que puede enriquecer instantáneamente el debate que está teniendo lugar en el aula. Si bien es cierto que en el aula el estudiantado puede trabajar con materiales analógicos e impresos, el empleo de dispositivos electrónicos como tabletas, ordenadores, etc. es útil para interactuar con el Campus Virtual, revisar los ejemplos ya vistos en clase o para buscar otros paralelos de intervenciones o gestión en otros bienes patrimoniales, acceder a información complementaria previamente compartida, etc.

- -Pensar en los materiales de forma dinámica y colaborativa. Realmente, los esquemas o guías almacenados en el Campus Virtual no eran apuntes, por lo que 
el alumnado debía sentirse libre para añadir comentarios, esquemas, ejemplos, datos, etc., todo aquello que cada persona consider ase relevante en su proceso de aprendizaje. Estos documentos constituían un material de partida para elaborar el conocimiento a demanda del alumnado, ya que podían utilizarse de forma individual o en grupo; dentro o fuera de clase o, incluso, en red. Su manejo suponía una implicación del alumnado en su propio proceso de aprendizaje, basándose en la planificación, la flexibilidad y la responsabilidad.

- En un primer momento, se propuso la elaboración de tres vídeos, por parte de cada grupo o individuo, relacionados con la aplicación de los contenidos teóricos y la presentación de casos concretos en las sesiones de prácticas de aula, los cuales se vieron reducidos a dos finalmente. El motivo principal se debió, en primer lugar, al alto número de presentaciones elaboradas y, en segundo lugar, a su duración. Esta última cuestión, dejó patente el interés e implicación del alumnado de tercer y cuarto curso en la realización de esta actividad.

Esta metodología docente fue sometida a la valoración no sólo por parte de las docentes teniendo en cuenta los resultados en relación a los indicadores establecidos sino también por parte del alumnado a través de una encuesta, como será explicado en el siguiente epígrafe.

\section{RESULTADOS}

De acuerdo con la convocatoria de proyectos de innovación docente de la Universidad de Oviedo, debían establecerse diferentes indicadores (Figura 1) para realizar una valoración final del proyecto solicitado. Tales indicadores debían detallar los instrumentos utilizados para recoger la información y, al menos, uno de ellos debía estar vinculado con el grado de satisfacción del alumnado que participase en el proyecto. Por tanto, se decidió elaborar una encuesta de satisfacción a partir de la cual extraer las conclusiones oportunas sobre esta innovación. Esta encuesta fue realizada de forma voluntaria y anónima, los resultados que mostramos a continuación pertenecen a 21 alumnos y alumnas de tercer y cuarto curso del Grado en Historia del Arte.

\begin{tabular}{|c|c|c|c|}
\hline \multicolumn{2}{|r|}{ Indicador } & Modo de evaluación & Rangos fijados y obtenidos \\
\hline 1 & $\begin{array}{l}\text { Encuesta de satisfacción, } \\
\text { exposición de dificultades y } \\
\text { propuestas de mejora }\end{array}$ & $\begin{array}{l}\text { La encuesta } \\
\text { cumplimentada de forá } \\
\text { voluntaria y anónima por las } \\
\text { alumnas y alumnos en el } \\
\text { antepenúltimo seminario } \\
\text { práctico de la asignatura }\end{array}$ & $\begin{array}{l}\text { Entre } 0,0 \text { y } 20 \% \text { bajo } \\
\text { Entre } 20 \% \text { y } 50 \% \text { aceptable } \\
\text { Entre } 50 \text { y } 70 \% \text { bueno } \\
\text { Obtenido: } 90,4 \%\end{array}$ \\
\hline 2 & $\begin{array}{l}\text { Número de alumnos } \\
\text { alumnas aprobados }\end{array}$ & $\begin{array}{lccc}\text { Porcentaje de } & \text { alumnas } & \text { y } \\
\text { alumnos que } & \text { superen la } \\
\text { puntuación de } 5 & & \end{array}$ & $\begin{array}{l}\text { Entre } 0,0 \text { y } 20 \% \text { bajo } \\
\text { Entre } 20 \% \text { y } 50 \% \text { aceptable } \\
\text { Entre } 50 \text { y } 70 \% \text { bueno } \\
\text { Obtenido: } 100 \%\end{array}$ \\
\hline 3 & $\begin{array}{l}\text { Mejora de los resultados } \\
\text { respecto a las calificaciones } \\
\text { de cursos anteriores }\end{array}$ & $\begin{array}{l}\text { Comparación } \text { de los } \\
\text { porcentajes de aprobados, } \\
\text { notables y sobresalientes } \\
\text { obtenidos en el curso en que se } \\
\text { aplique el proyecto respecto a } \\
\text { los anteriores }\end{array}$ & $\begin{array}{l}\text { Rango de mejora (en } \\
\text { comparación de los tantos por } \\
\text { ciento): } \\
\text { Entre } 0,0 \text { y } 20 \% \text { bajo } \\
\text { Entre } 20 \% \text { y } 50 \% \text { aceptable } \\
\text { Entre } 50 \text { y } 70 \% \text { bueno } \\
\text { Obtenido: } 15 \% \text { * }\end{array}$ \\
\hline
\end{tabular}

Figura 1. Indicadores establecidos para valorar el éxito de la consecución de este proyecto de innovación docente.

Fuente: Elaboración propia. 
La encuesta proporcionada al alumnado constaba de dos partes diferenciad as. En primer lugar, el alumnado contaba con un listado de diez afirmaciones - cinco de ellas relativas a la metodología de la Flipped Classroom y las cinco restantes relacionadas con el uso del vídeo como herramienta de creación y comunicación - con las que debía mostrar su acuerdo o desacuerdo a partir de a la Escala de Lickert de 1 "Muy en desacuerdo" a 5 "Muy de acuerdo". La segunda parte de la encuesta está formada por un total de cinco preguntas sobre su percepción personal de la metodología aplicada a la asignatura y las repercusiones que ésta tuvo en su proceso de aprendizaje.

En este sentido, los resultados obtenidos en las encuestas planteadas al grupo de alumnado voluntario fueron los siguientes:

Respecto al uso de los materiales para las clases, el $100 \%$ del alumnado concuerda en que haberlos leído facilita la comprensión del temario explicado en clase. Por otra parte, el 95,2\% está de acuerdo en que trabajar con los materiales antes de clase permite plantear dudas sobre el temario cuando se explica, hecho que favorece al proceso de enseñanza-aprendizaje en el aula. Asimismo, el 85,7\% del alumnado encuestado concuerda con que tener los materiales antes de las clases ayuda a no tener que concentrarse únicamente en tomar apuntes, al contrario que en el sistema tradicional, al igual que el $90,5 \%$ opina que poder utilizar dispositivos móviles conectados a la red permite acceder a información y favorece el debate en clase (Figura 2).
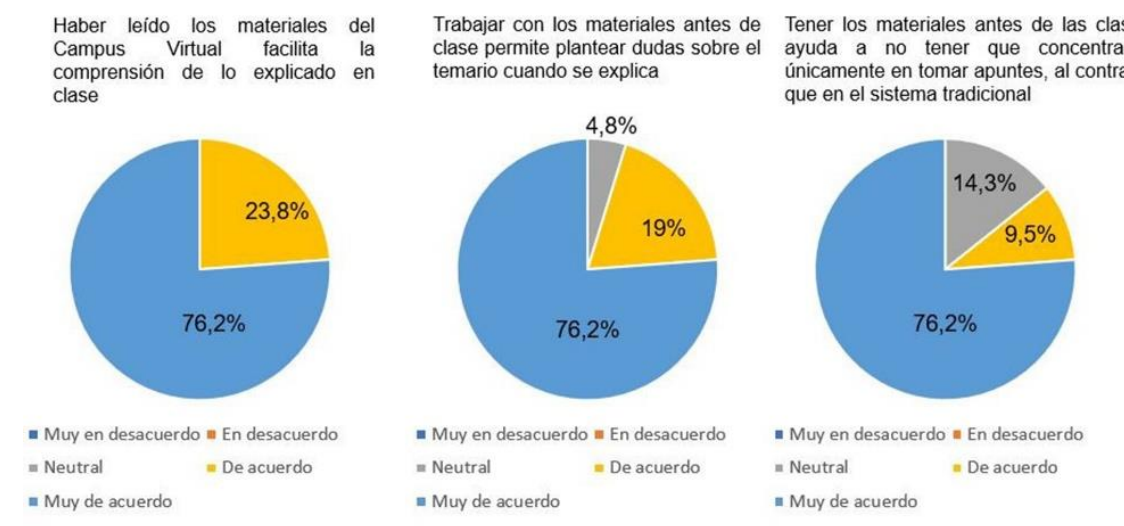
Poder utilizar dispositivos
conectados a la red permite
acceder a información y favorece el debate en clase

- Muy en desacuerdo " En desacuer - Muy de acuerdo

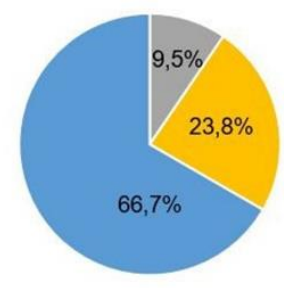

- Muy en desacuerdo $\mathbf{\text { En }}$ desacuerdo

- Neutral - De acuerdo - Muy de acuerdo

Figura 2. Resultados obtenidos en la encuesta de satisfacción del alumnado sobre la metodología aplicada.

Fuente: Elaboración propia

Por otra parte, el 85,7\% considera que esta metodología es efectiva en caso de no poder acudir a las sesiones presenciales (CEX y/o Pas), estando en desacuerdo únicamente el $4,8 \%$. Lo que refrenda y apoya la idea de la que partió el proyecto en este sentido (Figura 3). 
Esta metodología es efectiva en caso de no poder acudir a las sesiones presenciales (CEX y/o PAs)

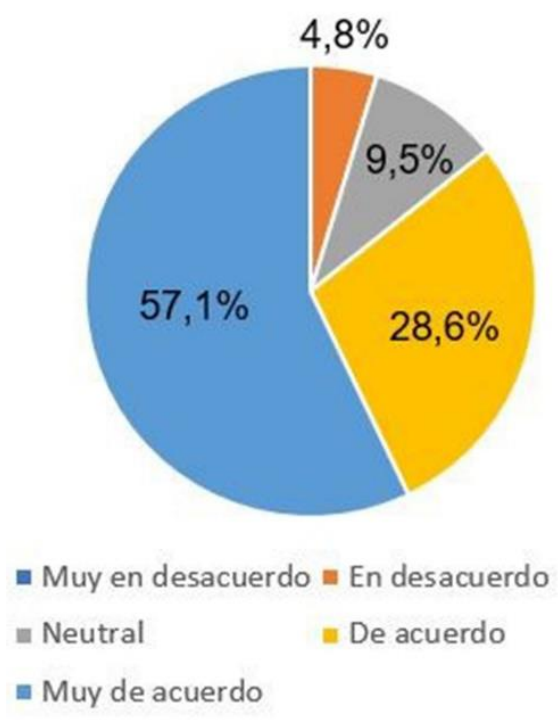

Figura 3. Resultados obtenidos en la encuesta de satisfacción del alumnado sobre la efectividad de la metodología en caso de no poder acudir a las sesiones presenciales. Fuente: Elaboración propia.

En lo que se refiere al uso del vídeo, el $81 \%$ del alumnado afirma que la realización del de esta actividad facilita el repaso y estudio constante de los contenidos de la asignatura. Este mismo porcentaje también opina que el uso de esta herramienta les ha servido para potenciar su capacidad de hacer presentaciones, exposiciones, etc. Partiendo del hecho de que las docentes consideramos que el manejo del vídeo y de las herramientas para llevarlo a cabo puede ser de utilidad de cara a un futuro laboral, nuestro alumnado respalda tal opinión en un $85,7 \%$ al estar de acuerdo con la siguiente afirmación: "La creación de vídeos es un método de enseñanza-aprendizaje de carácter práctico relacionado con una futura incorporación al mundo laboral (trabajo con las TICs, difusión de la disciplina, etc.)". Asimismo, el 90,4\% de nuestro alumnado está de acuerdo con que esta combinación metodológica ha hecho más atractiva y motivadora la asignatura, indicando el $85,7 \%$ que consideran que de esta manera han podido desarrollar su capacidad de trabajo y formación autónoma a través de la educación virtual (Figura 4). 


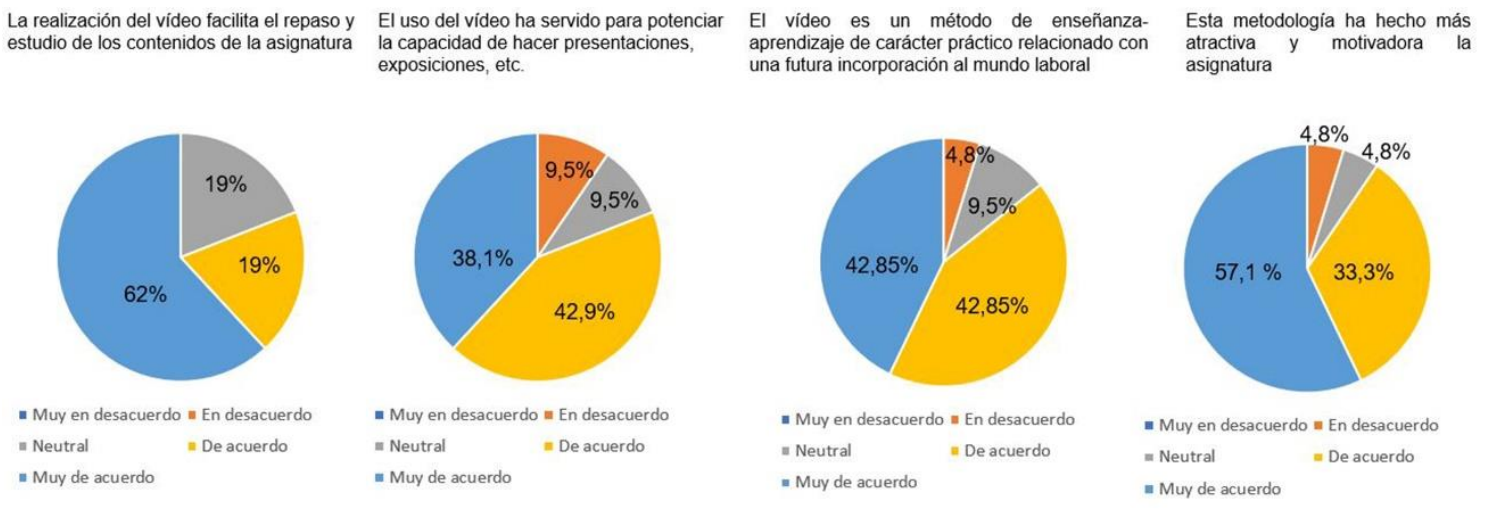

Figura 4. Resultados obtenidos en la encuesta de satisfacción del alumnado sobre el uso del vídeo como herramienta de aprendizaje.

Fuente: Elaboración propia

Por otra parte, el $100 \%$ de los alumnos y alumnas encuestadas han afirmado que han utilizado los materiales proporcionados a través del Campus Virtual en las propias clases y que elaboraron sus propios apuntes a partir de ellos de cara al examen de ambas asignaturas. Asimismo, el 90,4\% del alumnado ha concordado en que la elaboración de los vídeos ha hecho que el estudio de la asignatura fuese más constante, principalmente porque para su creación (aplicación del conocimiento adquirido) era necesario el repaso de los contenidos explicados en clase. Y, por otra parte, este mismo porcentaje de alumnado ha afirmado haberse sentido más implicada/o en la asignatura al realizar los diferentes vídeos (Figura 5).
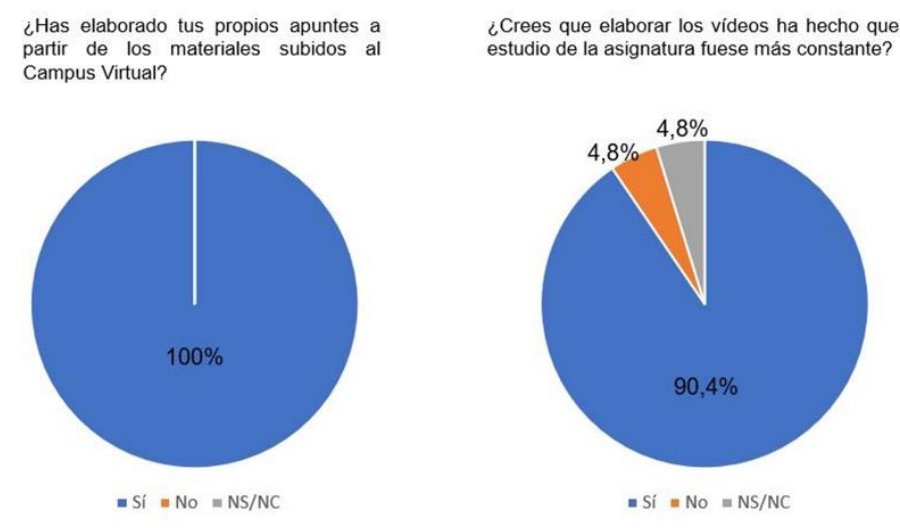
¿Te has sentido más implicada/o en la asignatura al
realizar los diferentes vídeos?

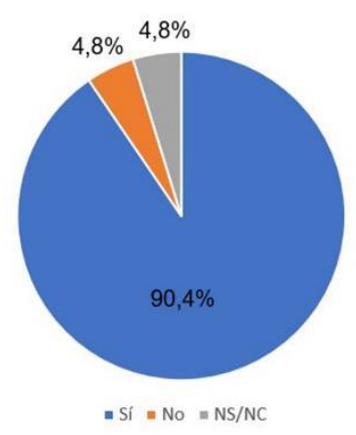

Figura 5. Resultados obtenidos en la encuesta de satisfacción del alumnado sobre la efectividad de la metodología en lo referido al proceso de aprendizaje.

Fuente: Elaboración propia

En cuanto al indicador de calificaciones obtenidas durante el curso 2019/2020, en el caso del grupo de tercer año, el cual ha cursado la asignatura de Historia de la Conservación y la Restauración del Patrimonio Histórico-Artístico, cabe señalar que el $11 \%$-correspondiente a dos, en número- de los suspensos corresponden a alu mnado de segunda convocatoria. Todo el alumnado de primera convocatoria superó el examen, siendo el notable la calificación obtenida en mayor proporción con un $44 \%$. En lo referente al grupo de cuarto curso en la asignatura de Gestión y Legislación del Patrimonio Histórico-Artístico, ya familiarizado con la innovación docente desde el curso anterior, todos los alumnos y alumnas superaron positivamente la evaluación, 
correspondiéndose con el notable el $56 \%$ de las calificaciones, seguidas por un $22 \%$ de sobresalientes obtenidos por el alumnado (Figura 6).
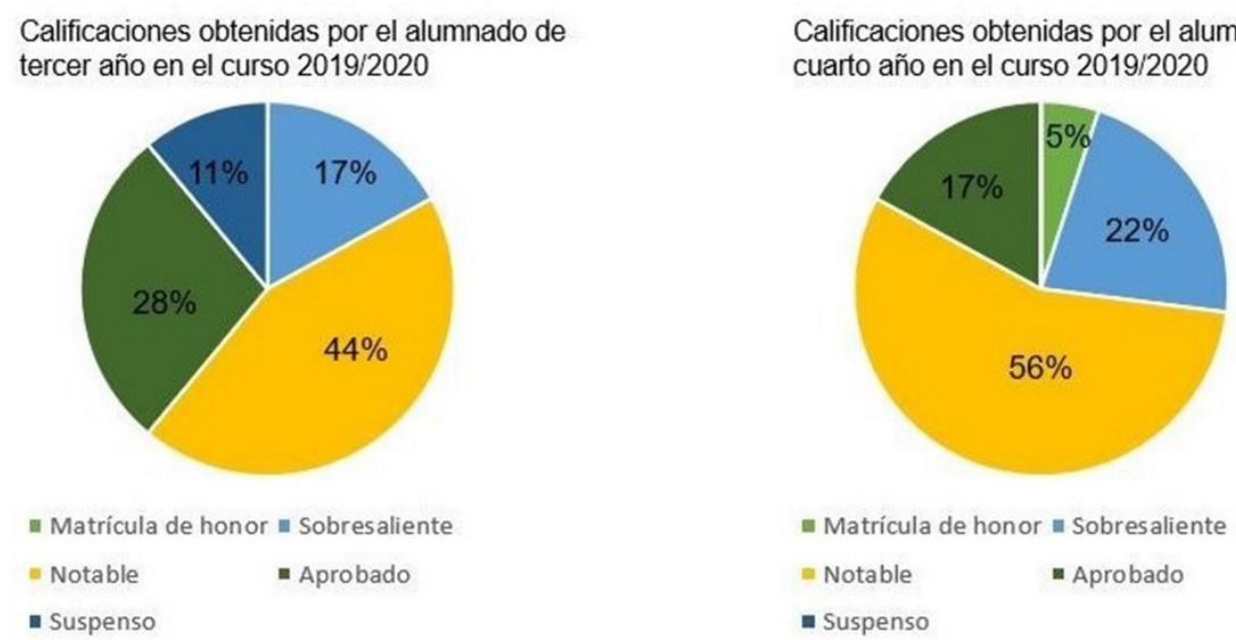

Figura 6. Calificaciones obtenidas por el alumnado de los cursos de tercer y cuarto año en el curso 2019/2020.

Fuente: Elaboración propia

Finalmente, con relación al tercer y último indicador consistente en la comparación entre las calificaciones obtenidas este curso académico y el anterior, en el que también fue aplicada la innovación docente como se ha dicho, se han percibido mejoras en ambas asignaturas (Figura 7).

En el caso de la asignatura de tercer curso Historia de la Conservación y Restauración del Patrimonio Histórico-Artístico que había centrado el proyecto del curso 2018/2019, se aprecia una mejora de los resultados de evaluación en los siguientes sentidos:

- $\quad$-El porcentaje de suspensos del curso pasado fue un $25 \%$, mientras que en este curso ha supuesto únicamente el $11 \%$. Sin embargo, debemos resaltar el hecho de que el alumnado de primera de convocatoria de este curso ha superado positivamente la evaluación final de la asignatura.

- La mejora en los porcentajes de aprobados, notables y sobresalientes, indicador que establecimos, supone un 15\%.

- Por su parte, en lo referente al alumnado de la asignatura Gestión y Legislación del Patrimonio Histórico-Artístico, de cuarto curso del grado, de nuevo apreciamos las siguientes mejoras:

- El porcentaje de alumnado que superó la asignatura el curso pasado fue un $91 \%$, mientras que en este curso ha sido del $100 \%$, suponiendo una mejora del $9 \%$ en este sentido.

- La mejora en los porcentajes de aprobados, notables y sobresalientes supone, de nuevo, un $15 \%$, si tenemos en cuenta el descenso de los aprobados (1\%), el aumento de los notables (19\%), pero también el descenso de los sobresalientes (5\%). 
En este sentido, nos parece necesario llamar la atención sobre el hecho de que, a pesar de ser este 15\% un porcentaje de rango de mejora "bajo", ya se había aplicado la metodología de la clase inversa en el curso 2018/2019. Por ello, se considera que haber revisado y perfeccionado el método para este segundo proyecto de innovación ha mejorado los resultados indiscutiblemente.

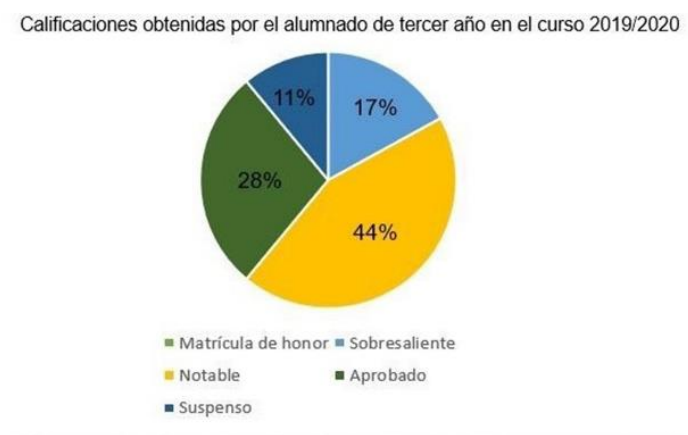

Calificaciones obtenidas por el alumnado de cuarto año en el curso 2019/2020

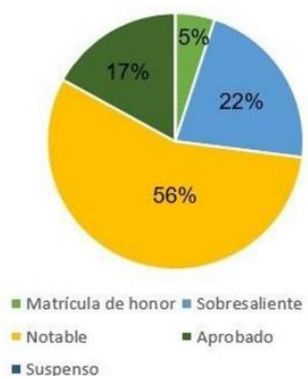

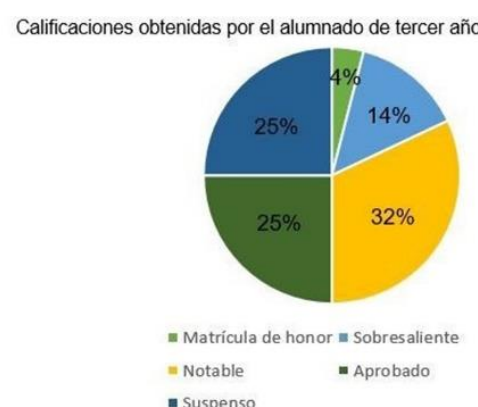

Calificaciones obtenidas por el alumnado de cuarto año en el curso 2018/2019

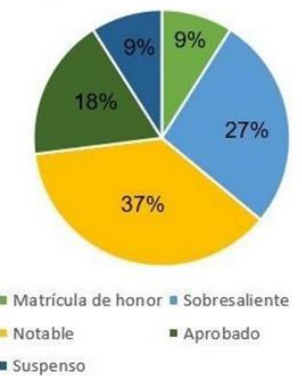

Figura 7. Comparativa de las calificaciones obtenidas por el alumnado de tercer y cuarto curso en las asignaturas incluidas en el proyecto durante los cursos 2018/2019 y 2019/2020.

Fuente: Elaboración propia

\subsection{Valoración conjunta del alumnado}

En lo referido a la aplicación de la clase inversa, se ha podido comprobar cómo el alumnado de tercer año, cuya relación con el método ha sido escasa a lo largo de los primeros cursos del Grado en Historia del Arte, ha respondido afirmativamente y, a pesar del esfuerzo que constituye adaptarse a una nueva metodología docente, la han valorado en el mismo sentido que las docentes y que sus compañeros y compañeras de cuarto año, quienes ya estaban familiarizados con ella debido a su participación en el proyecto anterior.

En esta línea, el alumnado de ambas asignaturas ha desarrollado su capacidad de trabajo y formación autónoma y entendido que esta metodología propicia la comprensión de los temas a tratar. De hecho, en una de las encuestas un/a alumno/a llega a afirmar: "Poder contar con el temario puntualmente, leyéndolo previamente antes de ir a clase, ayuda positivamente a que la clase no sea un conjunto de códigos indescifrables, el poder seguir la clase alternando explicación y lectura a un tiempo beneficia, en mi caso, la retentiva". Asimismo, también se ha valorado que esta metodología docente favoreciese una preparación más rápida de los apuntes finales y garantiza la optimización del tiempo en el aula. 
Por otra parte, nuestros alumnos y alumnas también han sido conscientes de que la Flipped Classroom permite mayor flexibilidad a la hora de trabajar, y han indicado a través de las encuestas que valoran que la responsabilidad de aprobar o no las asignaturas no recaiga exclusivamente en poder estar de forma física en el aula.

En lo relativo a la creación de vídeos, el punto de partida docente era favorecer la creatividad en el aula, y, a través de las encuestas, este planteamiento ha sido respaldado por el alumnado. Han considerado que esta herramienta ha favorecido esta competencia y ha dado lugar a un "nuevo tipo de seminarios (prácticas de aula) más atractivos e interesantes" a la vez que ha facilitado un estudio constante a través de un "actividad divertida, pero que obliga a investigar, releer los apuntes para entenderlos mejor".

Otra de las ideas principales de las docentes fue fomentar el trabajo colaborativo, y los resultados finales parecen haber ido incluso más allá de lo esperado. En este sentido, la colaboración en el aula no se ha reducido exclusivamente al trabajo en sí mismo, sino que se ha podido apreciar que la relación entre el alumnado de cada curso se ha visto favorecida. De hecho, esta cuestión ha sido reflejada también por los alumnos y alumnas en la encuesta de satisfacción, realizando las siguientes afirmaciones:

"Sea individual o en grupo, es interesante la forma de cada uno a la hora de plantear y presentar su trabajo. Esta forma de participación ha ayudado a comprender no sólo mejor las prácticas sino también ha favorecido una convivencia más llevadera y mayor respeto "entre nosotros", ayuda a la distensión y aleja de una competitividad insana".

"La puesta en común con la gente de clase me ha unido más a ellos".

"Ha facilitado que el trabajo en grupo sea más llevadero, solidario, participativo y que la preparación de la práctica sea diferente".

En cuanto a la relación enseñanza-aprendizaje, el alumnado ha manifestado que esta combinación metodológica hace que "se sienta más integrado" en la asignatura e, incluso, han valorado que se les haya obligado a "salir de la zona de confort" (refiriéndose a las tradicionales exposiciones en PowerPoint), puesto que además de haber podido aplicar los conocimientos adquiridos en las sesiones teóricas y prácticas, son conscientes de que han adquirido nuevas competencias, especialmente en relación a lo que denominan "informática".

\section{CONCLUSIONES}

La propuesta de esta iniciativa metodológica supone seguir profundizando en las diversas posibilidades que ofrece la innovación docente en el ámbito universitario, la Universidad de Oviedo, la Facultad de Filosofía y Letras y en el Área específica de Historia del Arte, siempre en relación con el sistema de Bolonia y el aprendizaje por competencias, para conseguir alcanzar un nuevo planteamiento docente.

El haber solicitado un proyecto de innovación el curso 2018/2019 con los resultados obtenidos supuso un impulso y un estímulo para las docentes y un importante punto de partida para seguir explorando nuevas posibilidades de 
innovación docente, en este caso introduciendo una nueva herramienta, como es el vídeo, que permitiese un mayor desarrollo de las competencias de nuestro alumnado.

La experiencia adquirida en el desarrollo de sendos proyectos confirmó la necesidad de animar a nuestros alumnos y alumnas a desarrollar una participación más activa en el trabajo sistemático con las nuevas herramientas que tienen a su disposición sumadas a las ya aplicadas. Asimismo, se ha podido comprobar que el fomento de la creatividad en las aulas no se opone en absoluto a la rigurosidad que se espera en el estudio, sino que, por el contrario, anima al alumnado a sentirse más involucrado en su propio proceso de aprendizaje.

Finalmente, y teniendo en cuenta las opiniones de nuestros alumnos y alumnas, se consideró necesario repensar para futuros proyectos la carga de trabajo asignada y redimensionarla de forma que se ajuste a su realidad académica. Si bien es verdad que la carga de trabajo puede aumentar para el alumnado al igual que para el profesorado con la metodología de la clase invertida, en el caso de la elaboración de los vídeos esta carga, junto con la de otras asignaturas, ha recaído eminentemente sobre el alumnado.

\section{REFERENCIAS}

Bergmann, J. \& Sams, A. (2014). A Flipped learning. Gateaway to student engagement. International Society for Technology in Education World. Washington, USA: Recuperado de https://books.google.es/books?id=hnQqBgAAQBAJ\&printsec=frontcover\&hl=es $\# \mathrm{v}=$ onepage\&q\&f=false

Herrera Batista, M.A. (2009). "Disponibilidad, uso y apropiación de las tecnologías por estudiantes universitarios en México: perspectivas para una incorporación innovadora". Revista Iberoamericana de Educación. Recuperado de https://www.researchgate.net/publication/28262955 Disponibilidad uso y apro piacion de las tecnologias por estudiantes universitarios en Mexico perspe ctivas para una incorporacion innovadora

Rutherford, S. Flipping the classroom - written case study. Recuperado de https://www.cardiff.ac.uk/learning-hub/view/flipping-the-classroom

Salinas, J. (2004). Innovación docente y uso de las TIC en la enseñanza universitaria. Revista de Universidad y Sociedad del Conocimiento (RUSC), 1(1) DOI: http://dx.doi.org/10.7238/rusc.v1i1.228

Touron, J. y Santiago, R. (2014) "El modelo Flipped Learning y el desarrollo del talento en la escuela". Revista de educación (368), pp. 196-208. Recuperado de https://www.mecd.gob.es/dctm/revista-de-educacion/articulos368/el-modeloflipped-learning-y-el-desarrollo-del-talento-en-laescuela.pdf ?documentld=0901e72b81e9f56f

Trujillo, J. M. (2011). Comunicación, innovación, educación y gestión del conocimiento en torno al uso del podcast en la educación superior. Revista de Universidad y Sociedad del Conocimiento (RUSC). Recuperado de http://rusc.uoc.edu/ojs/index.php/rusc/article/view/v8n2-trujillo/v8n2-trujillo 